\title{
Proceedings of the Scottish Otolaryngological Society Meeting, 13 May 2010, Dunkeld, Scotland, UK
}

Patient benefit from bone-anchored hearing aids in a Scottish rural setting

R Srivastava, F Ahsan, W McKerrow

From the Raigmore Hospital, Inverness, Scotland

Objective

To quantify the health benefit experienced by patients with bone-anchored hearing aids (BAHAs) living in a Scottish rural setting.

\section{Methods}

We designed a retrospective, cross-sectional, postal survey using the Glasgow Benefit Inventory questionnaire. The setting was the district general hospital in Inverness, Scotland. We studied 68 patients who had undergone BAHA implantation between 1997 and 2009. The intervention of interest was hearing rehabilitation using a BAHA, and the assessed outcome was the benefit obtained by patients from BAHA implantation, compared with other otolaryngological procedures.

Results

Of the 68 patients, we excluded 17 for various reasons. We received 44 responses from the remaining 51 patients, giving a response rate of 86 per cent. The female to male ratio was $2.1: 1$ and the mean patient age was 58.5 years. The median Glasgow Benefit Inventory score was +33.3 .

\section{Conclusion}

Patients' quality of life improved as a result of BAHA implantation, as measured by the Glasgow Benefit Inventory.

Scottish Audit of Head and Neck Cancer 1999-2001: laryngeal cancer

L Melia, S A Savage, K MacKenzie

From the Glasgow Royal Infirmary, Scotland

Objective

To determine the demography, treatment and survival outcomes of laryngeal cancer patients included in the Scottish Audit of Head and Neck Cancer 1999-2001.

Method

This was a comprehensive, multi-centre, prospective study of all newly registered laryngeal cancer patients in Scotland, from September 1999 to August 2001. Patients were identified from pathology records, then cross-checked using information from multi-disciplinary team meetings, surgical clinics, radiotherapy centres and the Information and Statistics Division Scotland.

Results

A total of 1910 new head and neck cancer patients were registered and followed up for 18 months. A total of 606 new laryngeal cancer cases were recorded; 26.9 per cent of these cases were from Scotland's most deprived areas, while 44.3 per cent presented with stage III or IV disease. The overall disease-specific five-year survival rate was 62.9 per cent.

\section{Discussion}

There have been recent changes in the diagnosis and treatment of laryngeal cancer, most notably endoscopic resection for early disease and organ preservation in more advanced disease. Further prospective studies of current treatment are required.

Assessment of paediatric pinna growth from frequency of hearing aid ear mould changes

G Dahanyake, S Ali, D Veitch

From the Royal Aberdeen Children's Hospital, Scotland

\section{Introduction and aims}

The human pinna continues to grow throughout a person's life. This study aimed to assess the growth rate of the pinna in the early years of life, and to determine when the peak growth rate occurs. This was done by extrapolating data from the frequency of hearing aid ear mould changes in a group of paediatric hearing aid users.

\section{Method}

Retrospective review of frequency of hearing aid ear mould changes in 100 children aged zero to 14 years who attended the Royal Aberdeen Children's Hospital hearing aid clinic between 2001 and 2010. This information was obtained from the computerised audiology database, on which data are collated prospectively. The frequency of hearing aid changes, due to ill-fitting ear moulds, was collected on a proforma for each individual patient, from zero to 14 years of age. 
Results

Pinna growth was fastest during the first year of life (10.24 changes per year). Between the ages of one and five years, the growth rate was moderate (4.25 to 1.58 changes per year). The growth rate was slower between the ages of six and nine years (1.21 to 0.83 changes per year), and then reached a plateau between the ages of 10 and 14 years (0.72-0.66 changes per year).

\section{Conclusion}

The growth rate of the human pinna is fastest in the first year of life, and reaches a plateau around the age of 10 years. These findings may have clinical significance when planning pinna reconstruction in children with congenital anomalies.

\section{Meatoplasty for intractable chronic otitis externa \\ A Chin, P Wardrop \\ From the Crosshouse Hospital, Kilmarnock, Scotland}

\section{Problem}

Chronic otitis externa affects 2 per cent of the population. Despite frequent clinic visits, aural toileting and topical treatment, some intractable cases do not respond to conservative treatment.

\section{Method}

We identified 27 patients who had undergone meatoplasty procedures, 21 for chronic otitis externa. These 21 patients were sent a Glasgow Benefit Inventory questionnaire and a visual analogue scale (VAS) tool in order to assess their symptoms.

Results

The response rate was 71 per cent (15 patients, 20 ears). The mean total Glasgow Benefit Inventory score was 44.81. The mean VAS symptom score was 0.8 (out of $10)$. Five patients had required bilateral meatoplasties. Fourteen patients reported no ear infections postoperatively. Two patients stated they would not have the operation in the other ear. However, all 15 patients stated they would recommend the operation to a friend. The mean duration from operation to questionnaire completion was 23 months.

\section{Conclusion}

In these patients with chronic otitis externa, meatoplasty was well tolerated and effective. This procedure should be considered for chronic otitis externa patients with intractable symptoms.

Liesegang diffusion of silver nitrate during cautery for epistaxis: a potential mechanism of cartilage damage

D Walker, D Baring, D MacLellan, B Bingham

From the Southern General Hospital, Glasgow, Scotland
Background

Epistaxis is a common ENT problem. The most common intervention is nasal septum cautery using silver nitrate. Septal perforation is considered to develop following excessive cauterisation or bilateral simultaneous cauterisation. This study examined the effect of silver nitrate on nasal septal cartilage, in order to elucidate the development of septal perforation.

\section{Methods}

We used bare nasal septal cartilage discarded during septal surgery. Four septal specimens were subjected to cautery using 75 per cent silver nitrate cautery sticks for lengthening periods of time. Specimens were then examined histologically to assess damage.

\section{Results}

As the silver nitrate stick was applied to the septal cartilage for a longer time period, the silver nitrate diffused through the septal cartilage to an increasing depth. This diffusion showed bands, a feature consistent with Liesegang diffusion. The actual tissue cauterisation burn was limited to the surface and did not penetrate into the cartilage. Whilst there was no evidence of tissue damage within the cartilage, this was difficult to assess in this tissue, being acellular. However, we did observe evidence of silver diffusion through the cartilage, indicating the possibility of tissue damage.

\section{Discussion}

Whilst our model was incomplete, in that it involved silver nitrate cautery directly onto the cartilage, it did show evidence of Liesegang diffusion of silver nitrate through the cartilage to greater depths with increased application time. This phenomenon has been widely discussed in both mineralogy and materials science. Although the silver nitrate burn itself does not extend deeper than the surface, it is the precipitation of silver, leading to oxidisation of any organic material in the area, which could lead to deeper tissue damage. A perforation may arise from surface devascularisation due to the chemical tissue reaction. However, this reaction occurs very quickly. Prolonged application of silver nitrate will not increase the degree of surface cauterisation but, as we have demonstrated, it will certainly increase the diffusion of silver nitrate into the cartilage. Our findings raise the question of whether post-cautery septal perforation is due to surface devascularisation or to an effect of silver diffused into the cartilage.

Speech, swallowing and quality of life in surviving laryngectomy patients: the West of Scotland Laryngectomy Audit

S Robertson, J Yeo, C Dunnet, D Young, K MacKenzie

From the Glasgow Royal Infirmary, Scotland 


\section{Background}

The West of Scotland Laryngectomy Audit has collected one of the largest datasets in the world literature evaluating the functional outcome and quality of life of surviving laryngectomy patients.

\section{Methods}

Questionnaire packs were posted to all laryngectomy patients in the West of Scotland Managed Clinical Network. Packs contained the Voice Symptom Scale, M D Anderson Dysphagia Inventory and University of Washington Quality of Life questionnaires.

\section{Results}

Patients who underwent primary laryngectomy alone, without receiving radiotherapy, reported significantly better Voice Symptom Scale scores $(p=0.015)$ and M D Anderson Dysphagia Inventory scores $(p<$ $0.0001)$ than those who underwent salvage laryngectomy. Patients using tracheoesophageal voice reported significantly better Voice Symptom Scale scores than those using other modes of communication $(p<0.01)$.

\section{Conclusion}

Significantly better voice and swallowing outcomes were reported by laryngectomy patients who had not received radiotherapy. These findings have implications for pre-treatment counselling in the context of organ preservation strategies for advanced laryngeal cancer.

\section{Day-case surgery experience in Aberdeen}

J R Newton, J Dmetrichuk, R Jones, D Y Veitch

From the Aberdeen Royal Infirmary, Scotland

\section{Introduction}

Previous studies have shown that day-case surgery is both clinically effective and 'patient friendly'. Elective performance of otolaryngological surgery often enables minimal morbidity. We thus believe that such cases are especially suitable for day-case management.

\section{Method}

This was a retrospective study examining 50 patients who had undergone elective ENT surgical procedures. Day-case surgery rates, procedures, fasting times and patient-related outcome measures were recorded, within our teaching hospital's busy otolaryngology unit.

\section{Results}

Procedures were conducted as a day case in almost 65 per cent of patients. Over 90 per cent of patients were pre-assessed. The mean patient fasting time was around 15 hours. Over 90 per cent of patients were very satisfied with their day-case experience.
Discussion

Factors which may improve day-case surgery rates and overall clinical effectiveness are discussed, including an effective pre-admission clinic, protected beds and appropriate pharmacy support.

\author{
Variation in outcome assessment after pinnaplasty \\ L Fraser, H Kubba \\ From the Royal Hospital for Sick Children, Glasgow, Scotland
}

\section{Introduction}

Comparison between different pinnaplasty techniques is currently difficult, due to lack of conformity in the assessment criteria used. We aimed to explore current methods of pinnaplasty outcome assessment used by pinnaplasty surgeons in the West of Scotland region.

\section{Methods}

We performed a prospective questionnaire survey of 23 cross-specialty pinnaplasty surgeons within our region. These surgeons were e-mailed an open-ended, electronic questionnaire enquiring about their technique, caseload, important success criteria and methods of outcome assessment. A reminder e-mail was sent three weeks later. All data were collected electronically and analysed.

Results

Nineteen surgeons replied, giving a response rate of 82 per cent. The average number of pinnaplasty procedures performed per year was 11, and there was wide variety in the surgical technique used. Most surgeons rated patient satisfaction as the most important criterion of success, rather than other, more technical determinants of success. Respondents' success criteria are discussed. Most respondents preferred speaking to the patient and looking at their ears, in preference to objective measurements or photography. Only three surgeons (16 per cent) described using ear measurements to assess outcome.

\section{Conclusion}

Our results indicate a wide variety of pinnaplasty practice and pinnaplasty outcome assessment methods. It is currently unclear how best to measure success following pinnaplasty, and the various options are discussed. Although patient-reported satisfaction appears to be the most popular success criterion, it remains a heavily biased outcome measure which cannot be reliably used for comparison between studies. Further research is required to develop validated outcome measures, incorporating both objective and subjective factors, which would enable more standardised measurement of patient benefit after pinnaplasty surgery. Such outcome measures could be used to compare pinnaplasty research, and for audit purposes. 
Quantifying the Carhart effect in otosclerosis: can overclosure be relied upon to improve audiometric results?

\section{Fraser, P Wardrop}

From the Crosshouse Hospital, Kilmarnock, Scotland

\section{Introduction}

The Carhart effect was first described in the 1950s, following the recognition that bone conduction thresholds improved in patients with otosclerosis following successful fenestration surgery. Knowledge of the factors influencing overclosure may help to predict patient benefit from otosclerosis surgery and assist patient selection and counselling. Predicting overclosure becomes particularly important in patients who have bilateral hearing loss, in whom surgery must generally make hearing in the operated ear normal or better than the non-operated ear in order for the patient to perceive benefit. Often, closure of the air-bone gap alone is not enough to provide audiometric benefit in such patients. We aimed to assess the predictability and amount of overclosure which can be expected following laser stapedotomy.

\section{Methods}

A retrospective case-note review was performed of patients undergoing laser stapedotomy with bucket handle piston, performed by the senior author between 2001 and 2009. Data were obtained from pre- and post-operative audiograms. Data collected included pre- and post-operative air and bone conduction thresholds, and pre- and post-operative air-bone gap. We also noted the presence of a Carhart notch on the patient's pre-operative audiogram (defined as a $5 \mathrm{~dB}$ or greater difference in bone conduction between two successive frequencies).

\section{Results}

We identified 27 patients. These patients' mean air conduction threshold was $31.4 \mathrm{~dB}$ pre-operatively (range 20-48) and $7.9 \mathrm{~dB}$ post-operatively (range 0-48). Eighty-nine per cent had evidence of a Carhart notch pre-operatively. Of those patients with notches, 8 per cent were at $0.5 \mathrm{kHz}, 22$ per cent at $1 \mathrm{kHz}$ and 70 per cent at $2 \mathrm{kHz}$. The mean post-operative bone conduction gain (i.e. amount of overclosure) was $5.2 \mathrm{~dB}$ at $0.5 \mathrm{kHz}, 11.7 \mathrm{~dB}$ at $1 \mathrm{kHz}$ and $12 \mathrm{~dB}$ at $2 \mathrm{kHz}$. Overclosure of $10 \mathrm{~dB}$ or more occurred in 33 per cent of patients at $0.5 \mathrm{kHz}, 70$ per cent at $1 \mathrm{kHz}$ and 70 per cent at $2 \mathrm{kHz}$.

\section{Conclusion}

Ninety-six per cent of patients achieved overclosure of $10 \mathrm{~dB}$ or more for at least one bone conduction frequency. Overclosure can be relied upon to improve bone conduction in the majority of patients undergoing laser stapedotomy. Knowledge of its occurrence has important clinical implications, particularly in those with bilateral hearing loss, who may need to rely on overclosure to achieve audiometric benefit from otosclerosis surgery. 